\title{
OBTENCIÓN, ANÁLISIS Y SIMULACIONES DE LA MATRIZ INPUT-OUTPUT DE LA COMUNA DE PUNTA ARENAS
}

\author{
SERGIO SOZA- AMIGO* Y JOSÉ MARIPANI"
}

\begin{abstract}
RESUMEN
El objetivo de este trabajo es mostrar y analizar la matriz input- output de la Comuna de Punta Arenas elaborada para el año 2003. Así, se explican los argumentos básicos del modelo, la forma en que se construye y las principales características de la economía materia de estudio. De esta investigación se constata que el $47 \%$ de la producción está concentrada en los servicios, un 37\% en la industria y un 16\% en el sector primario, además, se determina que el PIB comunal es de aproximadamente M\$832.502 (millones de pesos de 2003). De igual forma, se estiman y analizan los multiplicadores parciales y totales de producción, distribución e ingreso, a partir de los cuales se puede concluir que existen muchos multiplicadores parciales que apuntan a la existencia de una alta y baja interrelación sectorial. Adicionalmente, del análisis de los distintos multiplicadores totales, se puede observar lo variado, y en algunos casos, lo articulados que son para ciertas ramas.
\end{abstract}

PALABRAS CLAVE: insumo-producto, modelo de demanda, modelo de oferta, multiplicadores.

Clasificación JEL C67; O18; R15 y Z00

THE INPUT-OUTPUT MATRIX OF THE PUNTA ARENAS CITY: DESCRIPTION, ANALYSIS AND SIMULATION

\begin{abstract}
The objective of the present work is to show and analyzes the input-output matrix for the Punta Arenas city built for the year 2003. Hence, the model basic arguments, way of building such matrix and the main characteristics of the economic subject of study are explained. From these, it is shown that $47 \%$ of production is concentrated in services, $37 \%$ in industry and $16 \%$ in the primary sector, besides, the county's GDP is found to be approximately M\$832.502 (million pesos at 2003). In the same way,

Académicos de la Universidad de Magallanes, Facultad de Ciencias Económicas y Jurídicas, Punta Arenas, Chile. e-mail: sergio.soza@umag.cl, jose.maripani@umag.cl.

* Se reconoce y agradece el apoyo del Fondo de la Innovación y Competitividad (FIC-Magallanes), proyecto "Matriz InsumoProducto para la Comuna de Punta Arenas" y, del Núcleo de la Iniciativa Científica Milenio "Ciencia Regional y Políticas Públicas".
\end{abstract}


the partial and total multipliers of production, distribution and income are estimated and analyzed. From these, can be inferred that many partial multipliers exist that point toward a high and low sectional interrelationship. Also, from the analysis of the different total multipliers, it can be observed how different they are in some cases, and the articulated that they are for some branches.

KEY WORDS: input-output, demand-side input-output model, supply-side input-output model, multipliers.

JEL Classification C67; O18; R15 and Z00

\section{INTRODUCCIÓN}

Se ha optado por emplear la metodología input-output, dado que entre sus particularidades están, por nombrar algunas: el análisis que se deriva de los tipos de interrelaciones que presentan las distintas actividades, los pagos por factores productivos que de estas emanan, las interrelaciones que existen entre la economía y el resto del mundo, los distintos efectos que se pueden obtener luego de alterar la demanda final o elementos que conforman los inputs primarios o valores añadidos, en fin, de ella se puede obtener una serie de utilidades que sirven para distintas interpretaciones y simulaciones, de ahí que sea importante entre otros aspectos, el contar con tablas que reflejen el quehacer de las distintas economías locales.

Para entender la estructura e información que se puede desprender de una TIO o MIP (tabla inputoutput o, matriz insumo-producto), una opción es partir de la base que toda economía, está compuesta por agentes de actividad, en este sentido, una MIP muestra cómo cada actividad se relaciona con el resto por medio de las compras hechas de insumos, trabajo, capital e importaciones, de igual forma, el sistema no estará cerrado si no se incorpora en él a quienes les den uso a los productos que se elaboren, es decir, la economía requiere para su funcionamiento de la presencia de una demanda, la cual se manifiesta con las compras que hacen otros agentes, tales como, los hogares (consumo doméstico), el gobierno (consumo público), la inversión (formación bruta de capital) y las exportaciones.

Por tanto y, en base de lo anterior, se tiene que toda MIP consta de tres submatrices principales, la de Consumos o Demandas Intermedias, la de Inputs Primarios y Recursos (IP) o Valores Añadidos (VA) $y$, finalmente la de Demanda Final (DF).
En lo que continúa, se muestra cómo se obtuvo la MIP-PA (matriz insumo- producto para la Comuna de Punta Arenas), se realiza un breve análisis de la misma y se simula el impacto que generaría en la economía un aumento del 1\% de los turistas llagados en 2008 a dicha comuna.

\section{OBTENCIÓN DE LA MIP PARA LA COMUNA DE PUNTA ARENAS (MIP-PA)}

Dado el costo, por un lado, de levantar información para lograr una matriz con todos sus involucrados, y por otro, el que existe una tabla inputoutput regional referida al año 1996, confeccionada inicialmente por el INE a 73 ramas de actividad y, posteriormente, rediseñada por MIDEPLAN en el año 2003 a 40, es que se utiliza en este trabajo el denominado "enfoque temporal" para obtenerla. Elegido éste, y luego de analizar las distintas opciones que permiten actualizar dicha matriz y llevarla a un nivel comunal, se consideró que las metodologías que más se adecuaban a lo que se realizaría era el método RAS y el Cross Entropy Method, optándose finalmente, por la utilización del método RAS, esto último, dado que Golan, Judge y Robinson (1994, pp. 548), sostienen que con los métodos indirectos, en concreto, con el Cross Entropy Method y el RAS, se llega a similares resultados.

En virtud de lo expuesto, se optó por la técnica RAS y además se consolidó su uso en función de las siguientes consideraciones específicas:

1. El método de la minimización de la entropía cruzada se recomienda cuando la solución de la actualización de la tabla pasa por incluir restricciones del tipo de igualdades y desigualdades lineales (Uriel et al., 2003, pp. 105), que no es el caso. 
2. El método es simple aunque rígido, dada la información que requiere para su solución (Rodríguez et al., 2005, pp. 387), sin embargo, es muy útil si se tiene la información referida a los vectores de borde, los cuales se corroboraron por medio de encuestas en este caso.

3. De acuerdo con la literatura y los múltiples ejercicios empíricos, se observa que entrega resultados más precisos al ser comparados éstos con los obtenidos mediante métodos directos (Malizia y Bond (1974), Round (1978), Pedreño (1986) y Álvarez (2001).

4. La técnica RAS, que en esencia es un método bi-proporcional sintético, es una buena técnica al momento de estimar flujos intersectoriales intermedios, utilizando como base una MIP e información exógena (Pulido y Fontela 1993).

\section{APLICACIÓN DEL MÉTODO RAS}

Para iniciar el uso de esta técnica -la que no se detallada dada la abundante información que existe sobre ella-, se reunió un conjunto de datos estadísticos provenientes de distintas fuentes, tales como: Banco Central de Chile, Servicio de Impuestos Internos, Aduanas, Encuesta Nacional Industrial Anual (ENIA), Chile Emprende, etcétera.

La matriz de partida (base) corresponde a la "matriz de coeficientes técnicos de la región de Magallanes y Antártica Chilena del año 1996" confeccionada por MIDEPLAN (revisar, "Aproximación a las Economías Regionales con Base en Aplicaciones Insumo-Producto"; diciembre de 2005, pp. 188). El paso siguiente, y en consideración a la información recopilada de las fuentes secundarias antes mencionadas, fue definir los denominados "vectores de borde" partiendo del año 1997, en términos de ventas, compras y producción. En este sentido, con la matriz base más la información de las fuentes secundarias, se obtuvo la "matriz de coeficientes técnicos de la comuna de Punta Arenas para el año 1997".

Una vez repetido el proceso anterior año a año, esto es, obtener cada matriz anual de coeficientes técnicos de la comuna, con un cálculo iterativo de aproximadamente 90 pasos en consideración al error cercano al cero por ciento que se especificó, se logró, finalmente, la matriz preliminar de coeficientes técnicos del año 2003.

Una vez definida la matriz de coeficientes técnicos preliminar de la comuna, se dio paso a la realización de encuestas a las diferentes actividades económicas determinadas en la muestra ${ }^{1}$, el organismo encargado de realizar dicha labor fue el INE-Magallanes ${ }^{2}$.

Para obtener la MIP-PA "final" se realiza un cruce de información en donde se contrastan, corroboran y ajustan los resultados de la matriz anterior con las encuestas tomadas más la consideración de las opiniones vertidas por algunas autoridades y expertos en relación a la economía que se estudia.

$\mathrm{Al}$ respecto, se debe indicar que del total original de encuestas a tomar (muestra de un $\mathbf{n}$ igual a 378 actividades), finalmente se consideraron 352 ya que fueron éstas las que pasaron y cumplieron el proceso de consistencia realizado por la misma institución, número más que suficiente para validar lo realizado.

Con respecto a la matriz final, se puede afirmar que la matriz resultante, está dentro de los resultados razonablemente esperados, es decir, es un proceso de investigación y diseño estadísticamente significativo, entregando una respuesta relativamente precisa de los tipos de interrelaciones que se dan en la comuna.

Desde otra perspectiva, si bien es cierto que al iterar año a año se produce un efecto distorsionador por el precio en cada una de las etapas, es sabido que el mismo proceso corrige en cierta medida esta posible desviación. En tal sentido se puede señalar que los resultados no se ven afectados por el fenómeno precio al determinarse las distintas matrices, pues tras emplear directamente

1 En este paso, se tomo como referencia el trabajo desarrollado por René Reyes y Juan C. Miranda en 1996.

2 Para la muestra se considera como base el directorio empresarial proporcionado por el INE-Magallanes, a partir del cual se establece el número de actividades a encuestar considerando la estratificación de cada sector económico, esto es, en base a la Clasificación Industrial Internacional Uniforme (CIIU). Se hace notar que el tamaño del directorio es de 6898 actividades económicas, con esta información se confeccionó una muestra estratificada por etapas con una varianza de muestra que se expreso como la probabilidad de ocurrencia $\left(s^{2}=0,09\right)$ y un error del $1,5 \%$, lo que significo encuestar por parte del INE-Magallanes a cerca de 400 actividades económicas 
la matriz de coeficientes técnicos de la región para el año 1996 y los vectores de borde del año 2003 sin pasar por los años anteriores, se observó que no existen diferencias significativas en los resultados lo que se explica básicamente por las características (estabilidad estructural) e importancia de la comuna en la región.

\section{INTERPRETACIÓN Y USO BÁSICO DE UNA MIP SIMPLIFICADA PARA PUNTA ARENAS}

La MIP que se presenta surge de una investigación financiada por el Gobierno Regional de Magallanes y la Antártica Chilena, donde su objetivo central es la determinación de las relaciones existentes entre las diferentes actividades económicas (productos y servicios) que se dan en la Comuna de Punta Arenas. De esta forma, la investigación da muestras de las relaciones de compra y venta de insumos entre los distintos actores de ésta economía.

Partiendo de una estratificación de sólo tres sectores (primario, secundario y terciario o de servicios; tabla 1 del anexo 1), se puede observar que el PIB comunal es de 832.502 millones de pesos del año 2003, por su parte, el Valor Bruto de la Producción (VBP), alcanzo los 1.587.116 millones.

De la MIP-PA, también se aprecia, por ejemplo, que el sector primario produjo 250.323 millones, el secundario y terciario por su parte, 586.837 y 749.957 millones, respectivamente. En el caso de la producción del sector terciario, se observa que, 14.152 millones fueron consumidos por el propio sector (99.697 y 203.831 por los sectores primarios y secundarios, respectivamente), lo que arroja un consumo intermedio total para la economía de 754.615 millones.

En referencia a la demanda final, se observa que más del 52\% (M \$436.473), tiene como origen, el sector de servicios, esto es, la economía en general, basa sus intercambios en este tipo de actividades, dejando un escaso $16 \%$ al sector primario, esto se puede recoger como una buena señal, ya que las economía desarrolladas, en general, presentan una estructura similar a la aquí observada, habida consideración del nivel de agregación efectuado ${ }^{3}$.

3 Al respecto puede leer Soza- Amigo y Ramos (2010) o, Soza (2007).
En resumen, se observa que más del $42 \%$ de las compras que se requieren para la producción, están centradas en el sector terciario y un 38\% en el industrial, dejando sólo un 19\% aproximadamente para el sector primario.

Además de lo anterior, la metodología io (inputoutput), también permite conocer los requerimientos directos de producción para cada rama, los cuales se obtienen a partir de la matriz de coeficientes técnicos (tabla 2 del anexo 1), su nombre obedece a que dichos coeficientes indican la relación técnica entre los requerimientos de insumo y producto, esto es, señalan si son vistos verticalmente, cuál es la función de producción de la rama que se analiza. Cada elemento de esta última matriz se designa genéricamente como $\mathbf{a}_{\mathbf{i j}}$ (con $\mathbf{a}_{\mathbf{i j}} \in \mathbf{A}$ ) y se obtiene de dividir los elementos que forman la matriz de consumos intermedios e IP respecto a su producción $\left(\mathbf{z}_{\mathrm{ij}} / \mathbf{x}_{\mathrm{j}}\right.$; donde $\mathbf{x}_{\mathbf{j}}$ es el output de la rama j-ésima), en este caso, y centrándose en el sector terciario, ella nos indica que para producir una unidad de este sector, se requieren insumos por 0,0189 del sector primario, 0,1329 del secundario y 0,2745 del propio sector más, 0,5737 unidades de lo que corresponde al valor agregado.

Como un complemento a lo anterior, pero visto desde otro ángulo, Ghosh plantea una matriz de oferta, denominada matriz de distribución, la cual se obtiene en forma horizontal. Cada elemento se designa genéricamente como $\left(\mathbf{a}_{\mathrm{ij}}\right)$ y se calcula como $\mathbf{z}_{\mathrm{ij}} / \mathbf{x}_{\mathrm{i}}$, donde $\mathbf{x}_{\mathbf{i}}$ es el output de la rama $i-$ ésima. Por lo tanto, cada coeficiente de la matriz de distribución, mostrará la proporción en términos monetarios que emplea la rama de la fila i-ésima, y que se destina a cada una de las otras ramas o a la demanda final. Con esta nueva forma de plantear el problema, se da paso a que los inputs primarios (trabajo o capital empleado en la producción de esa j-ésima rama) sean las nuevas variables exógenas y no la demanda final, como en el caso de la matriz de coeficientes técnicos. De esta forma, los modelos de demanda (Leontief) y oferta (Ghosh) permitirían conocer cómo los cambios en ellas afectan a las distintas funciones de producción y distribución, ya que ahora se puede trabajar con dos variables exógenas (demanda final e inputs primarios), lo que le daría a los usos que se pueden obtener de las tablas mayor flexibilidad e interpretación. Para el caso de la comuna de Punta Arenas (ver anexo 1, tabla 3), 
se puede observar que, el sector terciario basa su funcionamiento precisamente en un autoconsumo importante, esto es, sólo un 5\% de sus servicios tienen como destino la industria primaria, un $8 \%$ en la secundaria, estando el resto $(1 / 3)$ concentrado en su propio funcionamiento y prácticamente un $60 \%$ en el consumo de hogares.

Sin embargo y, aún cuando, la información que se deriva de una matriz de coeficientes técnicos o de distribución es muy importante, así como lo es el saber cuáles son los requerimientos indirectos de las distintas actividades; también es, el indagar cómo una determinada actividad influye en forma indirecta al resto del sistema productivo. La información a que se hace mención, para la demanda y oferta, se puede obtener a partir de las matrices inversas de Leontief y de Ghosh, respectivamente.

Cada elemento de la matriz inversa de Leontief $\left(\mathbf{x}=\mathbf{B}=(\mathbf{I}-\mathbf{A})^{-\mathbf{1}}\right.$, con $\mathbf{b}_{\mathbf{i j}} \in \mathbf{B}$, donde $\mathbf{x}$ es la producción, $\mathbf{I}$ es una matriz identidad y $\mathbf{A}$, la matriz de coeficientes técnicos), expresará en cuanto se debe incrementar la producción de la rama i-ésima si se desea que aumente en una unidad la demanda final de la rama j-ésima. Por su parte, la suma en vertical, debe ser entendida como el aumento de la producción que debe realizar todo el sistema económico, a fin de satisfacer el requerimiento de una unidad por parte de la j-ésima rama (Rasmussen, pp. 127). Asimismo, la suma de una fila de la matriz inversa de Leontief, puede ser entendida como el aumento en la producción que debe realizar la i-ésima rama, cuando cada rama del sistema aumenta simultáneamente en una unidad su demanda final (Rasmussen, pp. 127). Para el caso que nos ocupa (en anexo 1, tabla 4), luego de revisar el caso del sector servicios, se observa que éste presenta un multiplicador en columna igual a 1,795 y a 2,074 en fila, por ejemplo, si el sector turismo, el cual está vinculado a los servicios, presenta un incremento en su demanda final de 100 millones de pesos, el sistema en su conjunto lo haría en MM\$ 179,5 (1,7950*100.000.000), estos requerimientos se distribuirían de la siguiente manera; 5,6 millones de pesos serían demandados por el sector primario, 30,2 millones por el secundario y 143,67 millones por la propia industria, de los cuales MM\$ 100 serían consumidos por la demanda final (consumo de hogares) y 43,67 millones serian solicitados como requerimientos intermedios para producir los 100 millones requeridos); como se aprecia, el incremento en la demanda final de éste sector, afecta al conjunto de la economía tanto en forma directa como indirecta. Si tal análisis se considera en filas y en el mismo sector servicios, su multiplicador $(2,0741)$, nos indicaría que, un incremento de una unidad en la demanda final de todos los sectores requiere un aumento de la producción de hasta 2,0741 unidades monetarias (0,3711 del sector primario; 0,2663 del secundario y 1,4367 del propio sector).

Por su parte, el valor de los elementos que forman la matriz inversa de distribución (en anexo 1, tabla 5), deben ser interpretados de la siguiente manera, por ejemplo, el elemento $\vec{b}_{\mathbf{2 ; 3}}=0,3821$ (relación entre el sector secundario y el terciario, indica que, si hay una reducción de un peso en el trabajo disponible del sector secundario (independientemente de las causas), entonces la producción del sector servicios se reducirá en 0,3821 (unidades monetarias, para este caso en concreto). Esta baja se presenta porque al reducirse el trabajo del sector secundario, se reducen los insumos del sector terciario, el cual depende de la producción del sector secundario.

La suma en filas de la matriz inversa de coeficientes de distribución mostrará el aporte que realiza cada rama para que aumente en una unidad los inputs primarios, por ello, se le conoce como el multiplicador de oferta o de inputs, de forma análoga, la suma en columnas indica en cuánto cambia la producción si se produce una modificación de una unidad en la oferta (inputs primarios) de cada una de las ramas que forman la MIP, lo cual según Pulido y Fontela (1993) sería equivalente a obtener el "multiplicador de una expansión uniforme de los inputs primarios". En el caso de Punta Arenas, y nuevamente centrándose en el sector terciario, si sus input primarios aumentaran en MM\$ 100 (digamos que aumentan los salarios en él), el sistema incrementaría la producción en MM\$1,769, los cuales se distribuirían de la siguiente manera; el sector primario vería incrementada su producción en $\mathrm{MM} \$ 12,4$, el secundario en $\mathrm{MM} \$ 20,84$ y el terciario en $\mathrm{MM} \$$ 143,67 (100 que son los requeridos por el propio sector y MM\$ 43,67 por el resto del sistema).

\section{ANÁLISIS DE IMPACTO}

La idea central de lo que continua, es analizar los multiplicadores de producción, distribución e in- 
Grafico 1: Multiplicadores de Producción para los 10 productos y servicios principales.

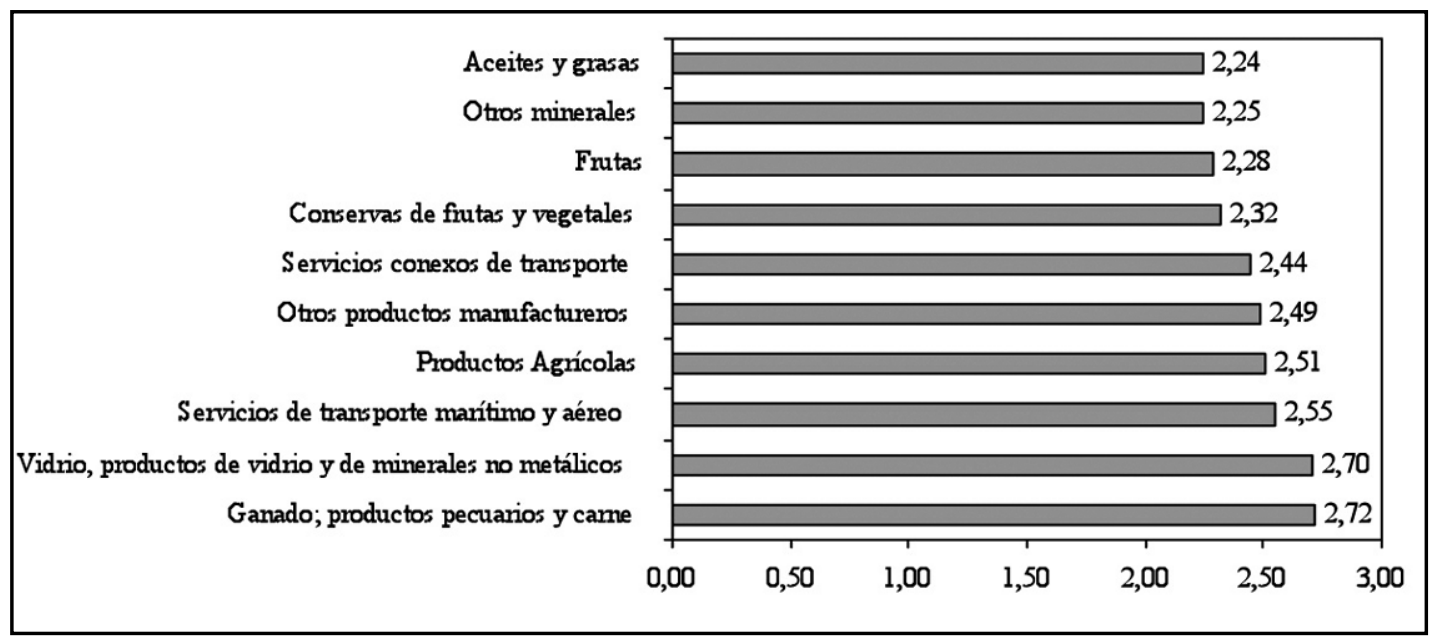

Fuente: Propia.

greso que se desprenden de la MIP-PA, información que se trabaja a un nivel de 40 productos y servicios de actividad (no confundir con ramas o sectores, se trata de productos y servicios).

Los multiplicadores de producción corresponden a la suma en vertical de la matriz inversa de Leontief, desde esta perspectiva se observa que dichos multiplicadores oscilan entre 1.30 (servicios de educación pública y privada) y 2,72 (ganado, productos pecuarios y carne). A su vez, lo productos con mayor impacto en términos de producción presentan unos multiplicadores que varían desde 2,24 (aceites y grasas) hasta 2,72 (animales; ver grafico 1).

Si la información anterior se conjuga con la del grafico 2 , se observa que, los 10 productos o servicios con mayor participación en el PIB comunal, generan el $86,54 \%$ de este, sin embargo, no se observa cierta correspondencia entre lo que cada producto o servicios cuando corresponda es capaz de generar, en términos de producción y su participación en el PIB, por ejemplo, para el caso del producto con mayor multiplicador, se observa que presenta un PIB que no la alcanza a situar dentro de las 10 actividades más importantes, de hecho esta en el lugar 22 , en contraposición, se aprecia que el producto que más aporta al PIB (productos químicos), presenta un multiplicador de producción bajo $(1,98)$, lo anterior, da un indicio del error que se puede cometer al fijar políticas económicas basadas en un criterio de participación del PIB y no en términos de lo que es capaz de inducir cada rama en términos dinámicos.
De igual forma, se observa que, en general, los mayores multiplicadores de producción estarían señalando que el desarrollo de la comuna de Punta Arenas, pasaría por inyectar recursos al sector secundario, esto quiere decir que, para generar una mayor producción se deben a su vez potenciar los sectores primarios (base), de esta forma se estaría impulsando en forma más articulada la producción local. Por otra parte, se observa un aspecto interesante de destacar, las actividades que se podrían vincular al turismo (transporte, comunicaciones, actividades de ocio, restaurantes, hoteles y comercio), no están presentes en términos de multiplicadores ni de PIB, luego esto sería una señal de que tal servicio no es relevante para el desarrollo de la comuna.

Por otra parte, los multiplicadores de producción de menor tamaño, en general, están presentes en los servicios, seguidos por el sector secundario y finalmente por el primario, esto en sí es, llamativo dado que para desarrollar la economía se deben potenciar las actividades pertenecientes al sector primario, los cuales estarían más articulados; y, además, es curioso, dado que la industria manufacturera (secundario), en general, presenta unos multiplicadores bajos (con la excepción de la rama vidrios, otros productos manufacturados y conservas), esto da una idea de lo poco articulado que es el entramado económico de este sector en ésta comuna, en términos de vínculos.

En referencia a los multiplicadores parciales, los cuales dan luz sobre las interrelaciones existentes 
Grafico 2: Productos y servicios que generan los mayores aportes al PIB comunal.

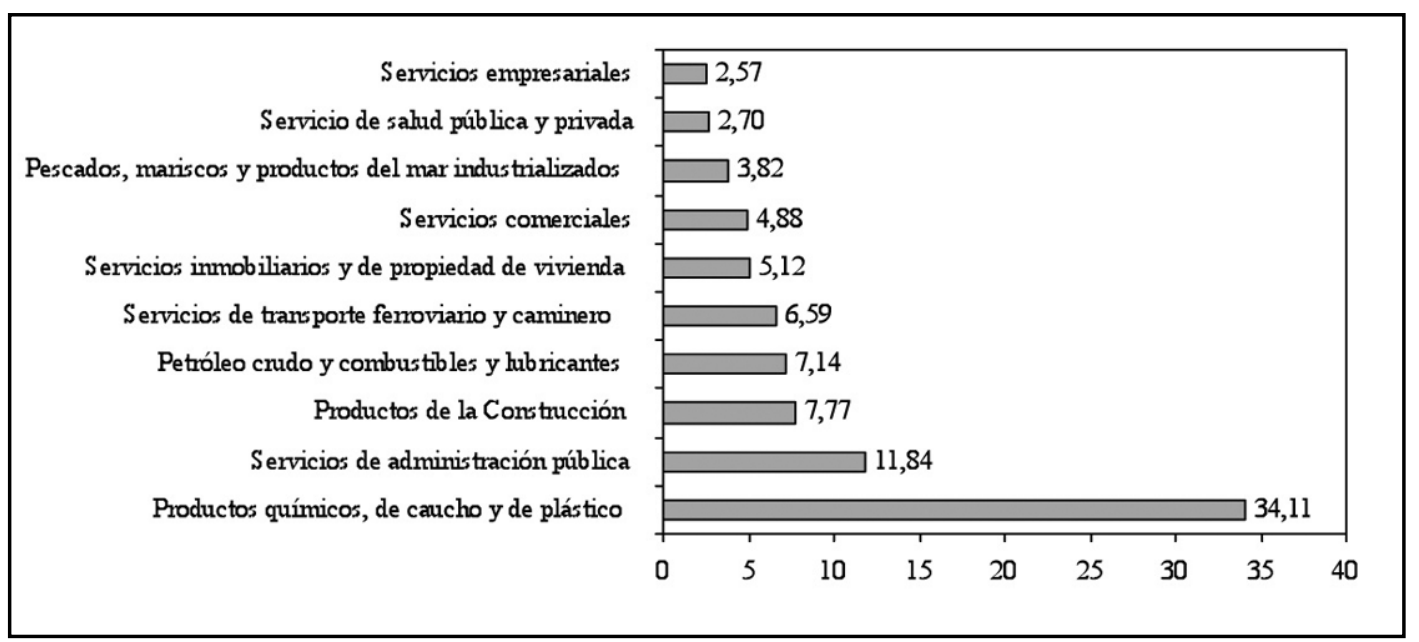

Fuente: Propia, basado en MIP-PA año 2003.

entre las distintas actividades, se aprecia que, los mayores valores están referidos a la propia industria, esto es, ganado, productos pecuarios y carne $(1,9037)$; productos químicos, de caucho y de plástico $(1,5805)$ y, de pescados, mariscos y productos del mar industrializados (1,4291), de igual forma, se observa que las mayores interrelaciones -exceptuando las de autoconsumo como las anteriores-, habrían estado vinculadas con la rama productos químicos, una interpretación de esto puede ser que, actividades tales como, la agricultura, frutas, ganado, verían afectada su producción si la industria química lo hace. Desde una perspectiva similar, se observa que existe un fuerte vínculo entre la rama transporte y productos silvícolas, pues su multiplicador es de 0,3089.

Desde una perspectiva más amplia, estos multiplicadores estarían señalando que las actividades "impulsoras" de la economía, esto es, aquellas que dan dinamismo a otras por la vía de la demanda de insumos, estarían concentradas en mayor número en el sector de los servicios, sin embargo, las que causarían un mayor impacto (dado que presentan una mayor magnitud), en general, estarían asociadas a los productos primarios.

Un aspecto igualmente interesante de revisar, son los valores que se encuentran en la diagonal principal de la matriz inversa de Leontief, ello dado que ofrecen información relativa al esfuerzo productivo total que debe realizar un sector ante un aumento de una unidad de su demanda final, esto es importante de analizar, dado que, tal magnitud refleja lo que una rama puede solicitar indirectamente de su propio producto para su producción (autoconsumo). Si tal valor se acerca a la unidad, será indicativo de que requiere poco autoconsumo para su producción y lo contrario si se aleja de éste, en este sentido, una vez sumada la columna de un sector, se puede restar su valor de la diagonal, y así observar los efectos indirectos sobre el resto del sistema productivo, o bien, restar una unidad y evaluar todos los efectos indirectos que tiene tal actividad sobre el resto del sistema incluyendo los que tiene sobre sí mismo.

Los mayores efectos indirectos los generan en los productos: ganado, productos pecuarios y carne $(1,72)$; vidrio y minerales no metálicos $(1,70)$; transporte marítimo y aéreo $(1,55)$; agricultura $(1,50)$ y otros productos manufacturados $(1,48)$. Lo anterior se entiende cómo el efecto que genera indirectamente al resto del sistema económico el aumento de una unidad de la demanda final del producto o servicios que se analiza, por ejemplo, en el caso del ganado, productos pecuarios y carne, para satisfacer un aumento unitario de su demanda final, se requiere que el sistema productivo aumente en 1,72 veces su producción.

Por su parte, los productos y servicios con mayores repercusiones sobre el sistema económico, excluyendo su autoconsumo y requerimientos indirectos, son los del vidrio y minerales no metálicos $(1,68)$; transporte marítimo y aéreo $(1,53)$; agricultura $(1,50)$; otros productos manufacturados $(1,49)$ y frutas $(1,28)$. Similar al caso anterior, por cada 
Gráfico 3: Multiplicadores de Distribución para los 10 productos y servicios principales.

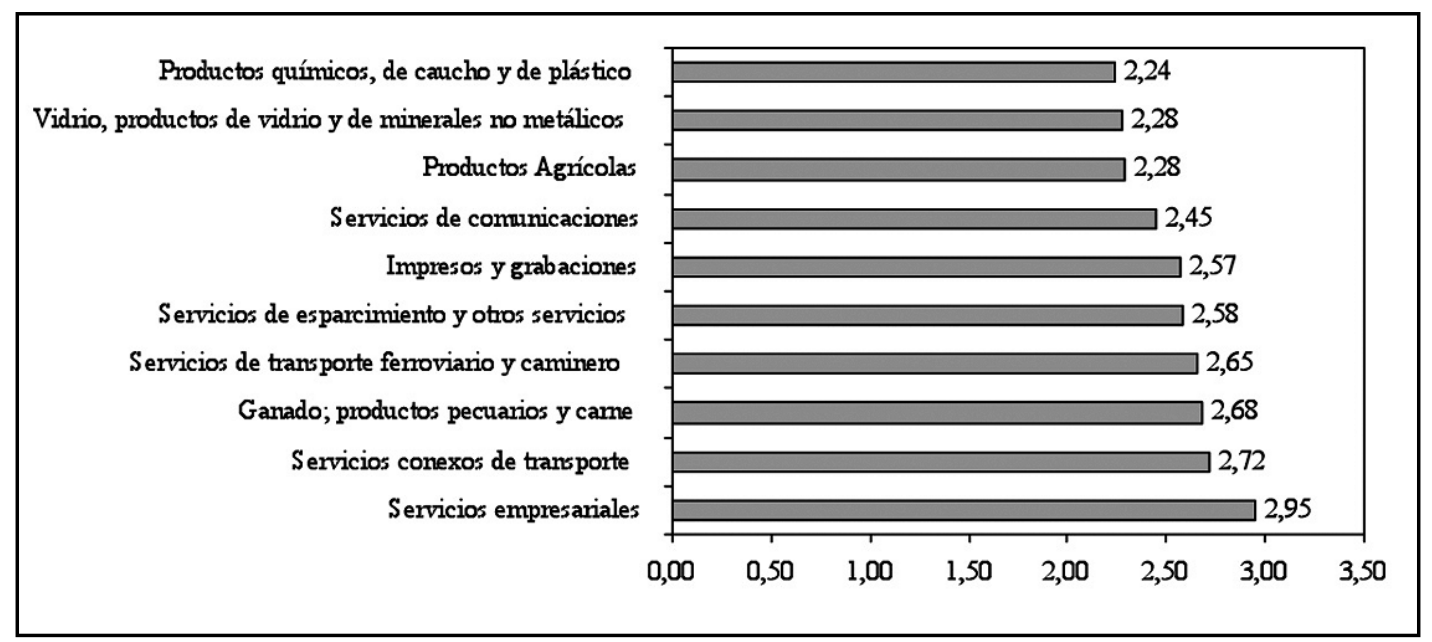

Fuente: Propia.

peso en que aumenta la demanda final de la rama vidrio y minerales no metálicos, esta requiere -en términos de aumento de la producción-, que el resto del sistema incremente su producción 1.68 veces.

Considerar los multiplicadores anteriores, es importante, puesto que la dinámica y nivel de desarrollo de una economía depende precisamente de los efectos indirectos, mientras mayores sean estos, es más fácil dinamizar la economía, ya que se puntualiza el esfuerzo pero se ramifican las consecuencias. En forma paralela, un entramado más articulado, esto es, que existan muchas relaciones indirectas es un paso previo para mayores niveles de desarrollo (Leontief, 1963, Carter, 1970, Forcell, 1988, Aroche-Reyes, 1996 y Cardero y Aroche 2008 y, Soza-Amigo y Aroca, 2010).

En base a los productos y servicios con mayores efectos indirectos, se observa que, en general, estos están presentes en los tres sectores, lo cual es beneficioso ya que si en la comuna se opta por intervenir en algunas de los productos o servicios con mayores efectos indirectos, pero que a su vez pertenezcan al sector primario, por consecuencia se estaría estimulando a los otros dos sectores, lo que a su vez motivarían la producción de la economía en su conjunto, como se ve, estos resultados ayudan al establecimiento de un derrotero que apunte a una planificación económica de la comuna.

En referencia a la matriz inversa de Ghosh, llama la atención las magnitudes extremas que se logran para el año 2003, su valor mínimo llega a 1,0004 (otros productos manufacturados) y su máximo a 2,9532 (servicios empresariales), tales multiplicadores dan cuenta de lo orientado que están algunas actividades a la entrega de insumos (en especial, los servicios) y lo concentrada que están otras (e.g., conservas de frutas y vegetales), en el sentido de que su actividad más bien depende de su propio autoconsumo.

Centrando este amplio abanico de valores sólo en los 10 principales multiplicadores de distribución, los que son representados en el gráfico 3, llama la atención, el efecto multiplicador que se desprende de la oferta de los servicios, esto es, las actividades orientadas a los servicios, no sólo tienen un gran impacto en términos distributivos, sino que además presenta un mayor número de actividades en esta condición para la economía Puntarenense (por ejemplo, transporte caminero, servicios conexos al transporte y comunicaciones), sin embargo, a su vez, presenta actividades con los menores impactos (administración pública, educación y salud). Lo interesante de las magnitudes anteriores, es el alto valor de sus efectos multiplicativos, nótese, que si los inputs primarios de los "servicios empresariales", se incrementasen en una unidad, esto es, por ejemplo, que sus rentas aumenten en $\$ 1$, las repercusiones del efecto total sobre la producción son de casi tres veces. En otras palabras, este valor representa la producción adicional que se produce en la economía, por cada unidad en que aumentan los inputs primarios ligados a los servicios empresariales. Por 
su parte, si en vez de aumentar en una unidad los input primarios, estos disminuyesen en tal valor, su valor indicará en cuanto disminuye la producción de la economía en su conjunto, dada la reducción en la producción que se origina debido a la disminución de las rentas o salarios del servicio en cuestión. Es decir, tal magnitud $(2,95)$ representa el posible impacto potencial que se originaría en la economía tras una variación de $\$ 1$, en los inputs primarios de la rama en cuestión.

Desde otra perspectiva, el análisis que sigue se centra en lo que realmente ayuda potencialmente al desarrollo local -en el sentido amplio y genérico del concepto-, esto es, los salarios y no en el aumento del producto, en este sentido, los multiplicadores de ingreso, dan una idea de la medida en que se ve afectada la producción en función de los primeros, habida consideración que, estos multiplicadores se centran en remuneraciones y no en el excedente bruto de explotación, dado que este último pertenece a los dueños del capital, los cuales, por lo general, no viven, en este caso, en la comuna, luego es esperable, que estas rentas por el uso del capital no queden en la comuna (Aroca, 2001, pp. 123 y Soza-Amigo y Aroca, 2010, pp. 92).

El gráfico 4, muestra los 10 principales multiplicadores de ingreso para la comuna de Punta Arenas, como se puede ver, el sector productos básicos del hierro, acero y metálicos, es el más significativo, su valor, expresado por cada $\$ 100$, nos indica que, por cada cien pesos producidos en tal sector, 83 son recibidos como salario, además, estos se descomponen de la siguiente manera, $\$ 73,91$ los reciben los trabajadores de la propia rama, $\$ 2,56$ que trabajan en otros minerales; $\$ 2,08$ los trabajadores de productos químicos, de caucho y de plástico; $\$ 1,12$ los remunerados del servicios de transporte caminero y; finalmente $\$ 3,21$ el resto de trabajadores.

Del gráfico 4, también se observa que los mayores efectos están vinculados nuevamente a actividades que se asocian a los servicios, de estos llama la tención el ligado a la educación dado que, si bien una parte importante del efecto se queda en el propio sector, tiene la particularidad de distribuir en forma uniforme el resto del impacto, esto es, su efecto restando su propia renta es muy disperso.

\section{SIMULACIÓN PARA EL SECTOR TURISMO}

Una de las mayores utilidades que se le suelen dar a las MIPs, es el derivado del uso de la matriz de coeficientes técnicos con sus respectivas funciones de producción y el de la inversa de Leontief y Ghosh, ello dado que las funciones de producción y distribución dan una visión del impacto directo que se puede obtener en una economía, luego de requerir determinados aumentos.

Aún cuando, el uso de las funciones anteriores son útiles, una visión o simulación más real,

Gráfico 4: Multiplicadores de Ingreso para los 10 productos y servicios principales.

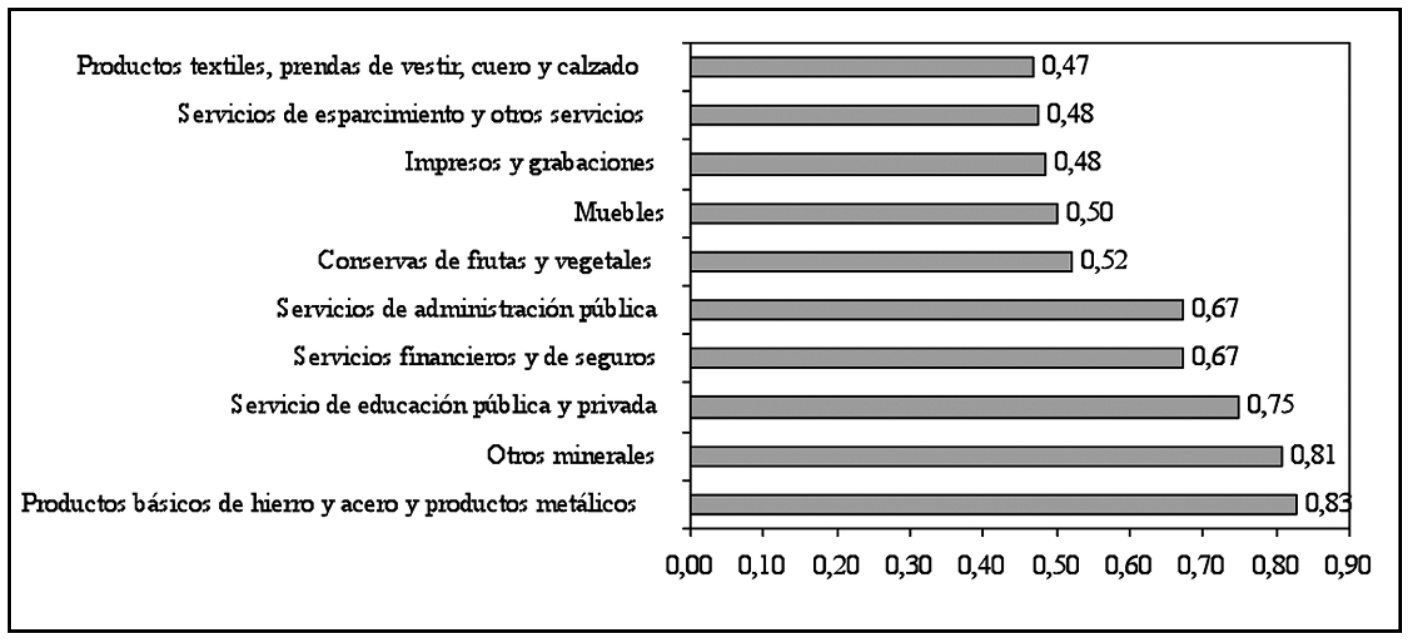

Fuente: Propia. 
se obtiene del uso del modelo de Leontief ( $\mathbf{x}=$ (IA) ${ }^{-1} \mathbf{y}$ ), donde $\mathbf{y}$ representa al vector de demanda final (consumo doméstico, de gobierno, formación bruta de capital y exportaciones) y, del modelo de oferta de Ghosh $\left(\mathbf{x}^{\mathbf{t}}=\mathbf{v}^{\mathbf{t}}(\mathbf{I}-\mathbf{B})^{-\mathbf{1}}\right)$, donde el superíndice $\mathbf{t}$, hace alusión a un vector transpuesto, $\mathbf{v}$ a los input primarios y $\mathbf{B}$ a la matriz de coeficientes de distribución. Lo anterior, obedece a que en tales modelos se cuantifican no sólo los efectos directos, sino que también los de autoconsumo e indirectos en términos de modificaciones para la demanda final e input primarios respectivamente, los cuales ya dan una primera visión de posibles repercusiones tras distintas simulaciones de shocks económicos.

Cómo una forma de aplicar los modelos anteriores, tómese como referencia práctica el siguiente ejemplo aplicado para el turismo en la comuna materia de estudio; primero, asuma que la estructura de gastos que se detalla en la Cuenta Satélite del Turismo para el año $2004^{4}$, no cambia, esto es, un turista desglosa su consumo en base a la Clasificación Internacional Industrial Uniforme (CIIU) de la siguiente manera; un 1,41\% lo gasta en combustible (comercio), un 18,69\% en servicios comerciales, un $29,19 \%$ en hoteles y alojamiento, un $25,28 \%$ en servicios de provisión de alimentación y bebidas (los dos últimos representan un 54,46\%), un $9,34 \%$ en servicios de transporte terrestre, un $5,38 \%$ en servicios de transporte marítimo y aéreo, un $1,22 \%$ en servicios conexos al transporte, un $1,80 \%$ en servicios de comunicaciones y, un 7,70\% en servicios de esparcimiento y otros; segundo, asuma que la información del último informe sobre turismo publicado por el $\mathrm{INE}^{5}$ se mantiene, esto es, la pernoctación promedio en la región es de 1,845 días (INE, 2009a, pp. 83), que la llegada de turistas que pernoctaron en la región fue de 201.235 personas (INE, 2009a, pp. 67; 2009b, pp. 32), que la pernoctación por llegados fue de 371.297 días (INE, 2009a, pp. 70, 2009b, pp. 32), que la tarifa promedio por alojamiento en la región ascendía a $\$ 74.478$ (INE, 2009a, pp. 85), que el ingreso

4 Ine-Magallanes: Investigación y Creación de la Cuenta Satélite de Turismo, XII región (2004). Revisada el 30 de diciembre de 2009 y, disponible en: <http://www.inemagallanes. cl/archivos/files/pdf/Estudios\%20Regionales/2009/6/ Cuenta\%20Satelite\%20de\%20Turismo\%20Informe\%20 Final\%202006.pdf>

5 INE (2009a). Turismo: Informe Anual 2008, pp. 106. promedio por pernoctación en la región fue de $\$ 45.215$ (INE, 2009a, pp. 87) y, que el gasto diario promedio total en Chile por concepto de turista extranjero fue de US\$60,9 (INE, 2009a, pp. 15), y finalmente que, según el INE (2009b) ${ }^{6}$, se tiene que durante el año 2008 a la comuna de Punta Arenas llegaron 109.292 turistas y pernoctaron 191.798 días (INE, 2009b, pp. 28 y 32), lo que indica que en promedio estuvieron 1,755 días en la comuna, además, según el INE (2009c) ${ }^{7}$ se tiene que el gasto promedio total fue de US $\$ 596,5$ por turista, luego, en base a lo expuesto, se pueden plantear, al menos, dos posibles escenarios:

1. Uno en que se asuma que efectivamente el turista gastó todo su presupuesto sólo en la comuna, esto es, los US\$596,5 en 1,755 días y, que los consumió de acuerdo al desglose presentado en la Cuenta Satélite de Turismo para la región del año 2004. Con respecto a este escenario, hay que indicar que es más acorde a lo que se observa en terreno, pues está en línea con la tarifa que se cobra en promedio por alojamiento en la región, la cual ascendía en esa fecha a $\$ 74.478$, quedando de este modo la diferencia para otros egresos asociados al quehacer cotidiano del turista (comida, bares, compra de souvenir, etcétera).

2. Asumir, un escenario más acorde al consumo diario, lo cual en la práctica significa aceptar una postura más pesimista dado lo bajo del consumo, en estas condiciones, se acepta que en promedio diariamente el turista gastó US $\$ 60,9$ y que permaneció en la comuna 1,755 días, es decir, cada turista gasta 106,8795 US $\$$, en la comuna mientras este en ella.

Asumir los supuestos anteriores, significa aceptar que por concepto de turismo, se gastó en forma directa lo que se indica en el cuadro 1 , dejando de lado los efectos indirectos e inducidos ${ }^{8}$. Sin embargo, aceptar este enfoque es en parte poco

6 INE (2009b). Estadísticas de Alojamiento Turístico Según Comuna: Año 2008, pp. 76.

7 INE (2009c). Comportamiento del Turidmo Receptivo Año 2008, pp. 91

8 El cual se asocia al incremento de los salarios por el aumento de la producción. 
Cuadro 1: Posibles Escenarios de Gasto Individual por Turismo

\begin{tabular}{|c|l|r|r|}
\hline \multirow{2}{*}{$\begin{array}{c}\text { Número } \\
\text { Rama }\end{array}$} & & \multicolumn{2}{|c|}{ Rama } \\
\cline { 2 - 3 } & & 3.498 & Optimista \\
\hline 28 & Combustible (venta) & 46.255 & 627 \\
\hline 28 & Servicios Comerciales & 134.795 & 8.288 \\
\hline 29 & Hoteles y Restaurantes & 23.115 & 24.152 \\
\hline 30 & Servicios de Transporte Terrestre & 13.312 & 4.142 \\
\hline 31 & Servicios de Transporte Marítimo y Aéreo & 3.014 & 2.385 \\
\hline 32 & Servicios Conexos al Transporte & 4.457 & 540 \\
\hline 33 & Servicios de Comunicaciones & 19.059 & 799 \\
\hline 40 & Servicios de Esparcimiento y Otros & 247.506 & 3.415 \\
\hline & Total Por Turista (diario) & & 44.348 \\
\hline
\end{tabular}

Fuente: Elaboración propia en base a datos del INE.

realista, pues deja de lado el consumo indirecto que se genera por estos consumos y el efecto inducido.

A modo de ilustrar el punto anterior, referido al efecto indirecto, asuma que se incrementa el consumo en restaurantes, ello trae aparejado algunos efectos tales como, un aumento en transporte debido al incremento en consumo, esto es, entre el centro de acopio y el restaurante, la edición de facturas y boletas, las posibles ampliaciones (muebles), el incremento en servicios comerciales asociados a este aumento, entre otros. Como se aprecia, existen una serie de consecuencias que quedan de lado.

Considerando lo anterior, lo que continua da respuesta, sobre la base de que se mantiene la estructura de consumo para el año 2003 y que ingresaron 109.292 turistas a la comuna durante el año 2008, a la pregunta ¿en cuánto aumenta la producción total sí, en ambos escenarios aumenta el número de turistas que llegan a la comuna, en un 1,0\% (1.093 personas), asumiendo que permanecen en la comuna 1,755 días $^{9}$ ?

Para el primer caso el gasto total, para los 1.093 turistas es de 271 millones de pesos de 2003 y para el segundo de 48 millones, por su parte, los efectos directos e indirectos, para esos consumos son respectivamente de 575 y 103 millones de pesos de 2003, es decir, la producción se incrementa en un $0,0362 \%$ y $0,0065 \%$ respectivamente. En

9 Otra alternativa, con análoga finalidad, podría ser el asumir que el número de visitantes se mantiene, pero se incrementa la pernoctación promedio, con el fin de analizar y evaluar cuáles son las posibles estrategias comunales que se deberían implementar para atraer, motivar e interesar a estos turistas a quedarse en la comuna un tiempo adicional y con ello incrementar la producción total. otras palabras, por cada peso gastado en turismo se produce un incremento de la producción de 2,13 pesos ( 1 por el propio servicio y 1,13 del resto del sistema económico).

En comparación a otros productos o servicios, se destaca que existen 12 actividadaes que reportan un aumento mayor que el registrado para el caso del turismo, como serían: ganado, productos pecuarios y carne (2,7213); vidrio, productos de vidrio y de minerales no metálicos (2,7045); servicios de transporte marítimo y aéreo $(2,5527)$; productos agrícolas $(2,5065)$; otros productos manufactureros $(2,4888)$; servicios conexos de transporte $(2,4435)$; conservas de frutas y vegetales $(2,3216)$; frutas $(2,2832)$; otros minerales (2,2461); aceites y grasas $(2,2412)$; servicios comerciales $(2,2113)$ y, petróleo crudo, combustibles y lubricantes $(2,1700)$ y, una que entrega retornos muy similares (servicios de hotelería y de restaurantes $(2,1225))$; luego, se puede afirmar que existen a lo menos 8 productos o servicios, ya que el resto está en parte vinculado al propio turismo, que podrían ser más significativos desde el punto de vista de un gasto eficiente versus el retorno que reportan a la economía.

Desde el punto de vista de la oferta, se podría asumir que dichos incrementos están asociados a los respectivos inputs primarios de cada rama (por ejemplo, aumento de las utilidades y/o salarios acompañados de impuestos, etcétera), en dicho escenario, se observa que el efecto de tal shock económico es mínimo, incluso existen 22 actividades con efectos multiplicativos mayores que el logrado por este concepto, el que alcanza una retribución de 1,8378 unidades monetarias, es decir, por cada peso que se incrementan los IP del turismo, la oferta global aumenta en 0,8378 unidades monetarias. 


\section{CONCLUSIONES}

La matriz insumo-producto de la comuna de Punta Arenas, es importante de analizar dado que tal comuna concentra la mayor actividad económica de la región de Magallanes y Antártica Chilena, en este sentido, dicha tabla no sólo proporciona una visión pormenorizada del tipo de interrelaciones que se dan al interior de la comuna, sino que además, señala cuáles son las rentas percibidas por cada actividad, en términos de cuánto de lo que se produce a nivel comunal se destina como insumo a otros productos $\mathrm{y}$, cuánto tiene como destino final el consumo directo de hogares.

Del análisis de los distintos multiplicadores, se puede observar lo variado que son y, en algunos casos, lo articulados que están ciertos productos y servicios, en este sentido, dicho análisis facilita la toma de decisiones, pues da una idea de la cuantía en que aumentaría, por ejemplo, la producción, si se alterase la demanda final de un determinado sector, o bien, cómo se vería afectada la producción, si se aumentase o disminuyesen los inputs primarios de una actividad, los que perfectamente podrían ser salarios o impuestos.

Lo anterior, es seguramente el mayor aporte que se puede desprender de la MIP-PA, pues en base a ella y dependiendo del tipo de objetivo que se proponga la autoridad, se podrían fijar distintas políticas que apunten a estimular la economía Puntarenense, que dada su condición, sin dudas repercutirá sobre la región en su conjunto. Estas políticas se podrían simular con mayor precisión, utilizando las herramientas propuestas en esta investigación.

De igual forma, se observa que existen muchos multiplicadores parciales, que apuntan a la existencia de una baja interrelación entre los productos y servicios que se elaboran, esto es, se aprecia un escaso consumo intermedio entre los sectores comunales, básicamente en el sector secundario (industria manufacturera), no así en los primarios y servicios.

Finalmente, la obtención de la MIP-PA, ha servido para realizar algunas simulaciones, de las cuales se desprenden los posibles efectos que se podrían originar en la economía, tras la aplicación de distintos shocks económicos y variados escenarios, en este sentido, la simulación ha sido referida al turismo, y los resultados indican que existen otras áreas en las cuáles se puede invertir con resultados que señalan mayores impactos globales.

\section{AGRADECIMIENTOS}

Los autores desean agradecer los comentarios y sugerencias vertidos por los académicos Osvaldo Pino (Universidad del Bío- Bío), René Reyes (Universidad de Los Lagos) y a Patricio Aroca (Universidad Católica del Norte). De igual modo, desean hacer presente su reconocimiento y apoyo al Gobierno Regional de Magallanes y la Antártica Chilena y, a la Universidad de Magallanes. Cualquier error $\mathrm{u}$ omisión es responsabilidad de los infrascritos, no comprometiendo por tanto a los anteriores.

\section{BIBLIOGRAFÍA}

ÁlVAREZ, R. 2001. Métodos de estimación indirecta de coeficientes input-ouput: una aplicación a la comarcalización de tablas. Trabajo de Investigación presentado en la Universidad de Oviedo.

AROCA, P. 2001. Impacts and Development in Local Economies Based on Mining: The case of the Chilien II región. Resources Policy. 27: 119-134

AROCHE-REYES, F. 1996. Important Coefficients and Structural Change: A multi-layer approach, Economic Systems Research, 8(3): 235-246.

BONET, J. 2000. La matriz insumo- producto del Caribe colombiano. Documentos de Trabajo, Centro de Estudios Económicos Regionales, Banco de La República de Cartagena de Indias, 15: 1-30.

CAÑADA, A. y I. TOLEDO 2001. Leontief y España: Una reflexión sobre las tablas input-output y su relevancia para la economía y los economistas españoles. Historia y Pensamiento Económico, 789:51-75.

CARDERO, M. E. y F. AROCHE 2008. Cambio Estructural Comandado por Apertura Comercial: El caso de la economía mexicana. Estudios Económicos, 23 (002): 203- 252

CARTER, A. 1970. Structural Change in the American Economy, Harvard University Press.

DORFMAN, R., P. SAMUELSON y R. SOLOW 1972. El Sistema Estático de Leontief. En su: Programación lineal y análisis económico. Segunda Edición, España, Editorial Aguilar, S. A., pp. 259.

FONTELA, E. y A. PULIDO 2005. Tendencias de la Investigación en el Análisis Input-Output. Revista Asturiana de Economía, 33:9-29. 
FORCELL, Ö. 1988. Experiences of Studying Changes in Input-Output Coefficients in Finland, en A. Smyshilav (comp.), Procedings of the Fourth IIASA Task Force Meeting on I-O model, Viena.

GOLAN, A., G. JUDGE y S. ROBINSON 1994. Rocovering Information From Incomplete or Partial Multisectoral Economic Data. The Review of Economics and Statistics, 76(2): 541-549.

LEONTIEF, W. 1985. Análisis Input-Output (1965). En su: Análisis Económico Input- Output. Segunda edición, España, Editorial Orbis, S. A., pp 226-227.

- 1963. The Structure of Development. Scientific American, (209): 148- 166.

- 1951. The Structure of American Economy, 19191939. New York, Oxford University Press.

- 1946. Exports, Imports, Domestics Output and Employment. Quarterly Journal of Economics, 58(2):290-313.

- 1944. Output, Employment, Consumption and Investment. Quarterly Journal of Economics, 60(2):171-193. - 1941. The Structure of American Economy, 19191929: An Empirical Application of Equilibrium Analysis. Harvard University Press.

- 1936. Quantitative Input and Output Relations in the Economic Systems of the United States. The Review of Economic Statistics, 18(3): 105-125.

KLEIN, L. 1953. On The Interpretation of Professor Leontief`s. Review of Economic Studies, 20:131-136.

MALIZIA, E. y D. BOND 1974. Empirical test of RAS method of interindustry coefficient adjustment. Journal of Regional Science, 14(3): 355-365.

MIDEPLAN, 2005. Aproximación a las Economías Regionales. Santiago, Chile, Ministerio de Planificación, pp. 211.

MUÑOZ, C. 2000. Las Tablas Input-Output del SEC-95. En su: Cuantas de la Nación. Segunda edición, España, Editorial Civitas, S. L., p 193.

PEDREÑO, A. 1986. Deducción de las tablas input-output: consideraciones críticas a través de la contrastación "survey-nonsurvey". Investigaciones Económicas, X (3): 579-599.

PINO, O. y J.C. PARRA 2006. Aplicación del Método Indirecto para la Obtencion de una Matriz Insumo-Producto año 2002 para VIII Región del Bío-Bío. Horizontes Empresariales, (5): 23-38.

PULIDO, A. y E. FONTELA 1993. Análisis Input-Output. Modelos Datos y Aplicaciones. España. Editorial Pirámide.

RASMUSSEN, P. N. 1956. Studies in Inter-Sectoral Relations. Amsterdam, North- Holland P. C.

REYES, R. y J.C. MIRANDA 1998. La matriz de Insumo Producto de Valdivia 1994. Propuesta metodológica para el análisis de las relaciones productivas de áreas menores. Universidad Austral de Chile, Valdivia, 200p.

RODRÍGUEZ, C., M. CARDENOTE y G. LLANES 2005. Estimación y actualización anual de matrices de contabilidad social: aplicación a la economía española para los años 1995 y 1998. Estadística Española, 47(159): 353-416.

ROUND, J.I. 1978. An interregional input-output approach to the evaluation of nonsurvey methods. Journal of Regional Science, 18(2): 179-195.

SADEI, 1988. Cuentas Regionales de Asturias 1985. Oviedo, España. Ed. Servicios de Publicaciones del Principado de Asturias. pp 19- 37, vol 2.

SOZA-AMIGO, S. y P. AROCA 2010. Oportunidades Pérdidas en Magallanes. Magallania, 38(2):89-101. Punta Arenas, Chile.

SOZA-AMIGO, S. y C. RAMOS 2011. La Agregación en las Tablas Input-Output: Una revisión desde la perspectiva de las ramas que no se unen. Revista de Economía Mundial, 28 (por aparecer).

SOZA, S. 2007. Análisis Estructural Input-Output: Antiguos problemas y nuevas soluciones. Tesis (Doctorado en Economía Aplicada). Oviedo, España, Universidad de Oviedo, Departamento de Economía Aplicada, 280 p.

STONE, R. 1962. A Social Accounting Matrix for 1960. A Programme for Growth. Chapman and Hall Ltd. London.

URIEL, E., J. FERRI y M. L. MOLTÓ 2003. La MCS-90 y la SAM-90 Evaluación de las diferencias. Estadística Española, 45 (152): 87-114.

TARANCÓN, M.A. 2003. Técnicas de Análisis Económico Input-Output. Alicante, Editorial Club Universitario, 269p.

THEIL, H. 1967. Economic and Information. Amsterdam, Nord-Holland, 488p. 


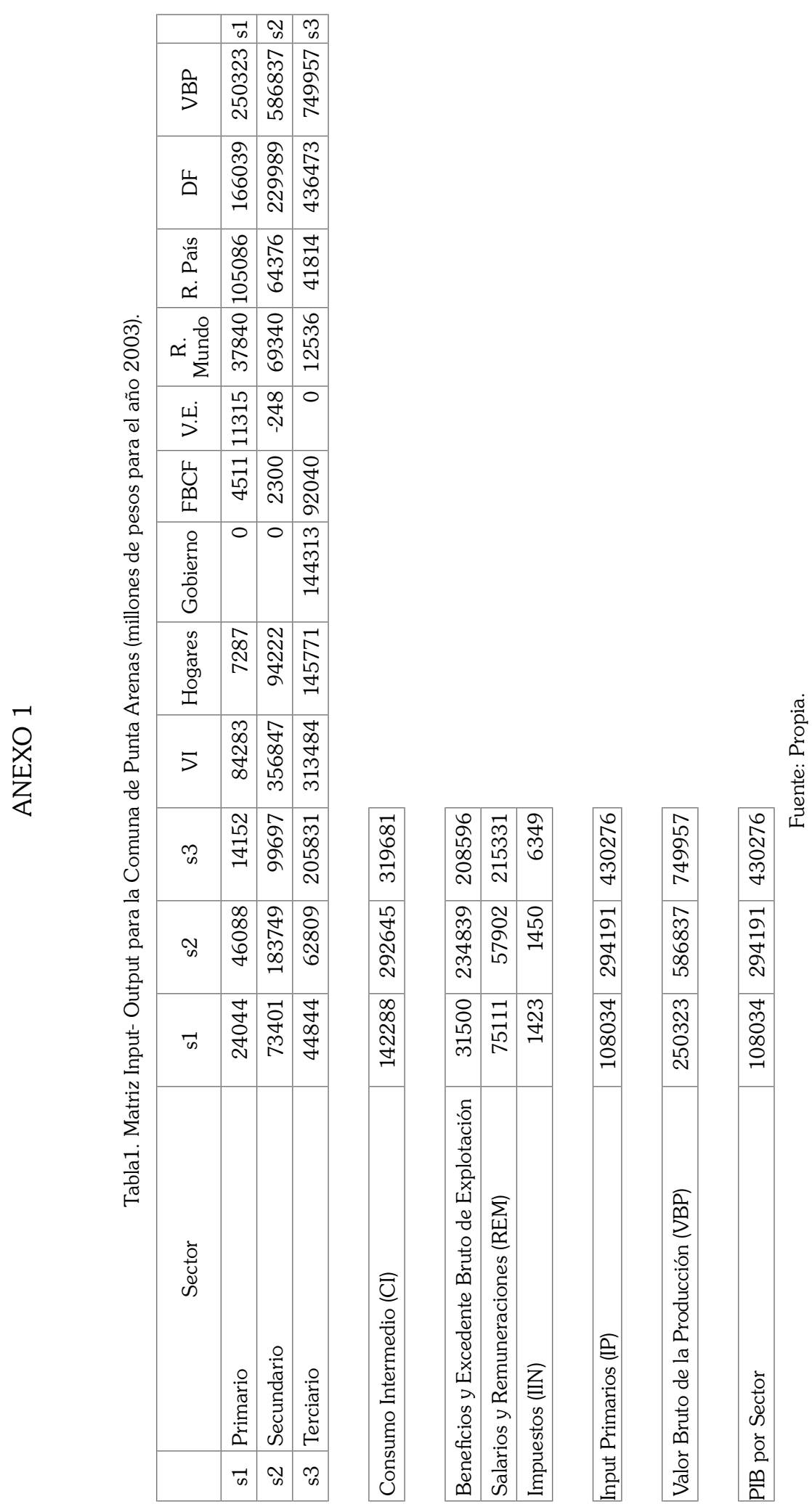


Tabla 2. Matriz de Coeficientes Técnicos para la Comuna de Punta Arenas.

\begin{tabular}{|r|l|r|r|r|}
\hline & \multicolumn{1}{|c|}{ Sector } & s1 & s2 & s3 \\
\hline s1 & Primario & 0.0961 & 0.0785 & 0.0189 \\
\hline s2 & Secundario & 0.2932 & 0.3131 & 0.1329 \\
\hline s3 & Terciario & 0.1791 & 0.1070 & 0.2745 \\
\hline
\end{tabular}

Consumo Intermedio (CI)

0.5684

0.4987

0.4263

\begin{tabular}{|l|r|r|r|}
\hline Beneficios y Excedente Bruto de Explotación & 0.1258 & 0.4002 & 0.2781 \\
\hline Salarios y Remuneraciones (REM) & 0.3001 & 0.0987 & 0.2871 \\
\hline Impuestos (IIN) & 0.0057 & 0.0025 & 0.085 \\
\hline
\end{tabular}

Inputs Primarios (IP)

0.4316

0.5013

0.5737

Valor Bruto de la Producción (VBP)

1.0000

1.0000

1.0000

Fuente: Propia

Tabla 3. Matriz de Coeficientes de Distribución para la Comuna de Punta Arenas.

\begin{tabular}{|l|l|r|r|r|r|r|r|}
\hline & Sector & s1 & s2 & s3 & VI & Total DF & VBP \\
\hline s1 & Primario & 0.0961 & 0.1841 & 0.0565 & 0.3367 & 0.6633 & 1.0000 \\
\hline s2 & Secundario & 0.1251 & 0.3131 & 0.1699 & 0.6081 & 0.3919 & 1.0000 \\
\hline s3 & Terciario & 0.0598 & 0.0837 & 0.2745 & 0.4180 & 0.5820 & 1.0000 \\
\hline
\end{tabular}

Fuente: Propia

Tabla 4. Matriz Inversa de Leontief.

\begin{tabular}{|l|l|r|r|r|r|}
\hline & Sector & $\mathrm{s} 1$ & $\mathrm{~s} 2$ & $\mathrm{~s} 3$ & Suma \\
\hline s1 & Primario & 1.1634 & 0.1418 & 0.0562 & 1.3614 \\
\hline s2 & Secundario & 0.5685 & 1.5679 & 0.3021 & 2.4385 \\
\hline s3 & Terciario & 0.3711 & 0.2663 & 1.4367 & 2.0741 \\
\hline Suma & 2.1030 & 1.9760 & 1.7950 & \\
\hline
\end{tabular}

Fuente: Propia.

Tabla 5. Matriz Inversa de Distribución o Ghosh para la Comuna de Punta Arenas.

\begin{tabular}{|l|l|r|r|r|r|}
\hline & Sector & s1 & s2 & s3 & 0.1685 \\
\hline s1 & Primario & 1.1634 & 0.3324 & 0.3860 & 1.6643 \\
\hline s2 & Secundario & 0.2425 & 1.5679 & 1.4367 & 2.1964 \\
\hline s3 & Terciario & 0.1239 & 0.2084 & 1.9912 \\
\hline Suma & 1.5298 & 2.1087 & 1.7690 \\
\hline
\end{tabular}

Fuente: Propia 


\section{ANEXO 2}

Formulaciones que determinan el tipo de igualdades para una MIP y encadenamiento según las distintas técnicas empleadas (hacia atrás $(\mathbf{B L})$ y delante $(\mathbf{F L}))^{10}$.

Ventas ínterindustriales $\left(\mathbf{z}_{\mathbf{i j}}\right)+$ demanda final $\left(\mathbf{c}_{\mathbf{i}}\right.$; consumo, ofd; resto de demanda final $)=$ ventas totales $\left(\mathbf{x}_{\mathbf{i}}\right)$

$\sum_{j=1}^{n} z_{i j}+\mathbf{c}_{i}+$ ofd $i=x_{i}$

Compras interindustriales $\left(\mathbf{z}_{\mathbf{i j}}\right)+$ valor agregado $\left(\mathbf{w}_{\mathbf{j}}\right.$ : salarios; $\mathbf{o v}_{\mathbf{j}}$ : resto del valor agregado)+ importaciones $\left(\mathbf{m}_{\mathbf{j}}\right)=$ salidas totales $\left(\mathbf{x}_{\mathbf{j}}\right)$

$\sum_{j=1}^{n} \mathbf{z}_{i j}+\mathbf{w}_{j}+\mathbf{o v} \mathbf{v}_{j}+\mathbf{m}_{j}=\mathbf{x}_{j}$

Matriz de Coeficientes Técnicos

$A=\left[\mathbf{a}_{\mathrm{ij}}=\frac{\mathbf{Z}_{\mathrm{ij}}}{\mathbf{x}_{\mathrm{j}}}\right]=\left[\begin{array}{cccc}\mathbf{a}_{11} & \mathbf{a}_{12} & \cdots & \mathbf{a}_{1 \mathrm{n}} \\ \vdots & \vdots & \ddots & \vdots \\ \mathbf{a}_{\mathrm{n} 1} & \mathbf{a}_{\mathrm{n} 2} & \cdots & \mathbf{a}_{\mathrm{nn}}\end{array}\right]$

Matriz Inversa de Leontief

$(I-A)^{-1}=\left[\mathbf{b}_{\mathrm{ij}}\right]=\left[\begin{array}{cccc}\mathbf{b}_{11} & \mathbf{b}_{12} & \cdots & \mathbf{b}_{1 \mathrm{n}} \\ \vdots & \vdots & \ddots & \vdots \\ \mathbf{b}_{\mathrm{n} 1} & \mathbf{b}_{\mathrm{n} 2} & \cdots & \mathbf{b}_{\mathrm{nn}}\end{array}\right]$

Multiplicador de Producción
Matriz de Coeficientes de Distribución

$$
\overrightarrow{\mathbf{A}}=\left[\overrightarrow{\mathbf{a}}_{\mathrm{ij}}=\frac{\mathbf{Z}_{\mathrm{ij}}}{\mathbf{x}_{\mathrm{i}}}\right]=\left[\begin{array}{cccc}
\overrightarrow{\mathbf{a}}_{11} & \overrightarrow{\mathbf{a}}_{12} & \cdots & \overrightarrow{\mathbf{a}}_{1 \mathrm{n}} \\
\vdots & \vdots & \ddots & \vdots \\
\overrightarrow{\mathbf{a}}_{\mathrm{n} 1} & \overrightarrow{\mathbf{a}}_{\mathrm{n} 2} & \cdots & \overrightarrow{\mathbf{a}}_{\mathrm{nn}}
\end{array}\right]
$$

Matriz Inversa de Ghosh

$$
\overrightarrow{\mathbf{B}}=(\mathbf{I}-\overrightarrow{\mathbf{A}})^{-1}=\left[\overrightarrow{\mathbf{b}}_{\mathrm{ij}}\right]=\left[\begin{array}{cccc}
\overrightarrow{\mathbf{b}}_{11} & \overrightarrow{\mathbf{b}}_{12} & \cdots & \overrightarrow{\mathbf{b}}_{1 \mathrm{n}} \\
\vdots & \vdots & \ddots & \vdots \\
\overrightarrow{\mathbf{b}}_{\mathrm{n} 1} & \overrightarrow{\mathbf{b}}_{\mathrm{n} 2} & \cdots & \overrightarrow{\mathbf{b}}_{\mathrm{nn}}
\end{array}\right]
$$

Multiplicador de Ingreso
$\mathbf{O}_{j}=\sum_{i=1}^{n} \mathbf{b}_{i j}$
$\mathbf{H}_{\mathrm{j}}=\sum_{\mathrm{i}=1}^{\mathrm{n}} \frac{\mathbf{w}_{\mathrm{i}}}{\mathbf{x}_{\mathrm{i}}} \mathbf{b}_{\mathrm{ij}}$ 